\title{
Índice glicêmico e carga glicêmica de dietas consumidas por indivíduos obesos
}

\author{
Glycemic index and glycemic load of diets \\ consumed by obese individuals
}

Helena Alves de Carvalho SAMPAIO ${ }^{1,4}$

Bruna Yhang da Costa SILVA2,4

Maria Olganê Dantas SABRY ${ }^{3,4}$

Paulo César de ALMEIDA

\section{RES U M O}

\section{Objetivo}

Avaliar o índice glicêmico e a carga glicêmica de dietas de indivíduos obesos.

\section{Métodos}

Trata-se de estudo retrospectivo, que analisou as fichas clínicas de 80 adultos obesos, acompanhados em um serviço privado localizado em Fortaleza, Ceará. Determinaram-se o índice glicêmico e a carga glicêmica da dieta. Para verificação da associação entre índice glicêmico e carga glicêmica, e dessas variáveis com a ingestão energética diária e com o índice de massa corporal dos indivíduos, utilizou-se o teste de correlação de Pearson. $O$ teste " $t$ " de Student foi utilizado para verificar diferenças entre os dois índices e o sexo. Em ambos os testes adotou-se $p<0,05$ como nível de significância.

\section{Resultados}

Verificou-se predomínio de índice glicêmico inadequado (moderado ou alto) no desjejum (82,9\%), no lanche vespertino $(60,0 \%)$ e no jantar (64,6\%). O índice glicêmico diário foi inadequado para 78,7\% do grupo e predominantemente moderado conforme a média encontrada $(59,23)$, porém menos inadequado que a carga glicêmica diária, que foi alta $(143,8)$, e pior, no sexo masculino. O índice de massa corporal do grupo, em geral e segundo o sexo, não foi associado com quaisquer dos dois índices. A ingestão energética diária associou-se apenas com a carga glicêmica, seja em geral ou considerando o sexo.

\section{Conclusão}

Os resultados apontam para uma maior importância da carga glicêmica na avaliação dietética desta clientela, sugerindo a inclusão de sua determinação na rotina de atendimento.

Termos de indexação: carga glicêmica; índice glicêmico; ingestão de alimentos; obesidade.

\footnotetext{
1 Universidade Estadual do Ceará, Curso de Graduação em Nutrição e dos Mestrados Acadêmicos em Saúde Pública e Ciências Fisiológicas. Fortaleza, CE, Brasil. Av. Paranjana, 1700, Campus do Itapery, 60740-903, Fortaleza, CE, Brasil. Correspondência para/Correspondence to: H.A.C. SAMPAIO.

2 Nutricionista. Fortaleza, CE, Brasil.

${ }^{3}$ Universidade Estadual do Ceará, Curso de Graduação em Nutrição. Fortaleza, CE, Brasil.

${ }^{4}$ Universidade Estadual do Ceará, Grupo de Pesquisa Nutrição e Doenças Crônico-Degenerativas. Fortaleza, CE, Brasil.
} 
616 | H.A.C. SAMPAIO et al.

\section{A B S T R A C T}

\section{Objective}

To evaluate the glycemic index and glycemic load of diets of obese individuals.

\section{Methods}

This is a retrospective study that analyzed the medical records of 80 obese adults attending a private health care service in Fortaleza, Ceará. The glycemic index and load of their diet was determined. The Pearson correlation test was used to verify if there was an association between glycemic index and glycemic load and of these variables with their daily energy intake and body mass index. The Student's " $t$ " test was used to verify the differences between the two indexes and gender. A significance level of $p<0.05$ was adopted for both tests.

\section{Results}

Inadequate (moderate or high) glycemic index prevailed at breakfast (82.9\%), afternoon snack (60.0\%) and dinner (64.6\%). The daily glycemic index was inadequate for $78.7 \%$ of the group and predominantly moderate according to the mean found (59.23), however it was less inadequate than the daily glycemic load which was high (143.8) and worse among males. The body mass index of the group, in general and according to gender, was not associated with any of the indices. Daily energy intake was associated only with glycemic load, both generally and taking gender into account.

\section{Conclusion}

The results point toward a higher glycemic load in the dietary assessment of obese individuals, suggesting that glycemic load should be routinely determined in this population.

Indexing terms: glycemic load; glycemic index; eating; obesity.

\section{N T R O D U ÇÃ O}

Os esforços para reduzir a prevalência de obesidade têm enfocado três variáveis em particular, sendo elas o aumento nos níveis diários de atividade física, a redução na ingestão total de gorduras e a limitação do consumo de açúcar e carboidratos rapidamente digeridos" ${ }^{1}$.

Nas décadas de 1960 e 1970 houve uma tendência de considerar os carboidratos como fator importante para o ganho de peso, desviando-se a atenção da gordura para o carboidrato, na hipótese de um nutriente atuar como principal promotor do excesso de ingestão energética e ganho de peso'.

Nas décadas de 1980 e 1990, inúmeros ensaios foram realizados para avaliar a resposta glicêmica produzida pelas diferentes fontes de carboidrato, principalmente, pelo amido, em diversos países².

Assim, mais recentemente, o alvo tem sido o papel do chamado índice glicêmico dos alimentos sobre a obesidade e as doenças a ela relacionadas ${ }^{3}$.
Define-se o índice glicêmico (IG) como a área sob uma curva de resposta à glicose, após o consumo de $50 \mathrm{~g}$ de carboidrato glicêmico (não incluídas as fibras) de um alimento teste, expressa como percentual de resposta para a mesma quantidade de carboidrato de um alimento padrão (pão branco ou glicose pura), ambos ingeridos pelo mesmo indivíduo 4 .

Sugere-se que as respostas hormonais, associadas às dietas com elevado índice glicêmico, como a hiperinsulinemia, promovem ganho de peso excessivo, provavelmente, por diminuir os níveis circulantes de combustíveis metabólicos, por estimular a fome e por favorecer a estocagem de gordura ${ }^{5}$.

Alguns estudiosos vêem o índice glicêmico como não fidedigno, visto que o mesmo não considera as porções reais consumidas por um indivíduo, sugerindo, assim, como melhor preditor de risco de doenças crônicas, entre as quais a obesidade, a carga glicêmica (CG) ${ }^{6}$, que inclui, concomitantemente, o IG do alimento e a quantidade de carboidratos disponíveis na porção de alimento consumida ${ }^{7}$. 
Entretanto, a comunidade científica não chegou ainda a um consenso quanto à realidade da conexão do índice glicêmico e da carga glicêmica com a obesidade.

Dessa forma, surgiu o interesse em avaliar o índice glicêmico e a carga glicêmica de dietas consumidas por obesos atendidos em um serviço privado de acompanhamento nutricional, assumindo a hipótese que a dieta dos mesmos possui índice glicêmico e/ou carga glicêmica inadequados.

Serviços especializados em atendimento nutricional, normalmente, elaboram dietas hipoenergéticas para perda de peso, apoiadas no hábito do indivíduo. Assim, caso haja um padrão de ingestão alimentar de alto IG/CG, existe a possibilidade de o mesmo ser reproduzido na dieta prescrita. Considerando as controvérsias da literatura, isso pode nada significar, ou pode comprometer todo o sucesso terapêutico. No Brasil, não há estudos publicados em literatura indexada avaliando a relação obesidade-índice glicêmico e/ou carga glicêmica dietética.

\section{MÉTODOS}

Estudo retrospectivo, baseado na análise documental de fichas clínicas de pacientes obesos de ambos os sexos, com idade maior ou igual a 20 anos, atendidos em um serviço privado de acompanhamento nutricional.

Considerando, aleatoriamente, os anos de 2003 e 2004, foram avaliadas as fichas clínicas de todos os pacientes que buscaram o serviço pela primeira vez para acompanhamento nutricional, num total de 420 indivíduos. Desses, 80 preencheram os critérios de inclusão: diagnóstico nutricional de obesidade (índice de massa corporal $\geq 30 \mathrm{~kg} / \mathrm{m}^{2}$, de acordo com a World Health Organization) ${ }^{8}$ e idade igual ou superior a 20 anos.

Entre os dados disponíveis nas fichas clínicas dos indivíduos que integraram a amostra, foram coletados: idade, sexo, índice de massa corporal e recordatório alimentar de ingestão habitual prévia ao início do acompanhamento nutricional. Deve-se ressaltar que todos os dados foram coletados por um único profissional, nutricionista, com especialização em nutrição clínica. O recordatório alimentar de ingestão habitual, segundo o protocolo do serviço, era obtido pela história alimentar, incluindo questionário de freqüência alimentar e recordatório alimentar de 24 horas, conforme Fisberg et al. ${ }^{9}$

Foi realizada uma caracterização geral dos pacientes, conforme o sexo, a idade média e faixa etária, bem como relativa ao grau de obesidade.

A obesidade foi categorizada, segundo o índice de massa corporal (IMC), em Obesidade grau I, quando o IMC esteve entre 30 e $34,99 \mathrm{~kg} / \mathrm{m}^{2}$, Obesidade grau II, entre 35 e $39,99 \mathrm{~kg} / \mathrm{m}^{2}$ e Obesidade grau III, quando $\geq 40 \mathrm{~kg} / \mathrm{m}^{2}$, critério de classificação estabelecido pela World Health Organization ${ }^{8}$.

O recordatório alimentar de ingestão habitual, referente a uma semana, continha o consumo de alimentos dos pacientes em medidas caseiras, transformadas em pesos, mediante padronização constante na tabela elaborada por Pinheiro et al. ${ }^{10}$. Na ausência do alimento na tabela, obteve-se a medida pela pesagem direta. Os recordatórios foram convertidos em consumo alimentar de um dia. A composição nutricional foi determinada com o auxílio do software "Sistema de Apoio à Decisão em Nutrição", versão 2.5a, da Universidade Federal de São Paulo, Escola Paulista de Medicina. A fim de conhecer os alimentos que mais freqüentemente interferiram no IG das dietas ingeridas, foram listados aqueles consumidos por, pelo menos, $50 \%$ dos indivíduos.

A determinação do Índice Glicêmico das refeições consumidas seguiu o protocolo proposto pela FAO/WHO Expert Consultation ${ }^{4}$ :

- Identificação do total de carboidrato glicêmico (em gramas) de cada alimento consumido por refeição;

- Determinação da proporção de carboidrato glicêmico de cada alimento em relação ao total de carboidrato glicêmico de cada refeição; 
- Localização do índice glicêmico de cada alimento (considerando a glicose como referência) em tabelas específicas (Foster-Powell et al. ${ }^{11}$; Brand-Miller et al. ${ }^{12}$ ). Para alimentos cujo índice glicêmico não foi estabelecido, utilizou-se o IG daqueles com semelhantes características;

- Determinação da contribuição de cada alimento ao IG da refeição, obtido a partir do produto do IG do alimento pela proporção de carboidrato glicêmico do mesmo em relação ao carboidrato glicêmico da refeição;

- Determinação do IG de cada refeição, pela soma dos valores obtidos no item anterior.

Determinado o índice glicêmico da refeição, esta foi categorizada como de baixo, médio ou alto índice glicêmico, mediante classificação de Brand-Miller et al. ${ }^{13}$, que definem: baixo IG, $\leq 55$; IG moderado, de 56 a 69 e alto IG, $\geq 70$.

Foi considerado adequado o consumo de refeições com baixo IG. As de moderado ou alto índice glicêmico foram vistas como inadequadas, seja para a prevenção ou para o controle da obesidade e das demais doenças crônicas. Esse critério de avaliação é sustentado por Wolever?

O índice glicêmico global da dieta foi obtido conforme Brand-Miller et al. ${ }^{13}$, por meio da média ponderada dos valores de IG de cada alimento, ou seja:

- Multiplicou-se o índice glicêmico de cada alimento pela proporção de seu carboidrato glicêmico em relação ao carboidrato glicêmico total da dieta;

- Realizou-se o somatório dos números obtidos;

- Categorizaram-se as dietas como de baixo, médio ou alto índice glicêmico, também com base na classificação de Brand-Miller et al. ${ }^{13}$ já referida.

Novamente, considerou-se adequado o consumo de uma dieta de baixo $\mathrm{GG}^{7}$.

A carga glicêmica diária foi determinada, conforme Lau et al. ${ }^{14}$, por meio do somatório dos produtos do carboidrato glicêmico de cada alimento, em gramas, pelo IG individual do mesmo, dividido por 100 .

Seguiu-se com a categorização das dietas como sendo de baixa, moderada ou alta carga glicêmica, conforme as mesmas tivessem CG menor que 80 , de 80 a 120 e maior que 120 , respectivamente ${ }^{15}$.

Consideraram-se adequadas as dietas de baixa CG, já que são capazes de manter os níveis glicêmicos estáveis, prevenindo hiperfagia posterior $^{6}$.

O IG e a CG diários foram confrontados com o IMC dos pacientes e com o valor energético total (VET) das dietas, no geral e considerando o sexo, na tentativa de verificar se valores mais elevados de IMC e/ou maior ingestão de energia estavam associados a um IG e/ou a uma CG diários também mais elevados. Para tanto foi aplicado o teste de correlação de Pearson, adotando-se $p<0,05$ como nível de significância. Foi verificada a diferença de IG e CG dietéticos diários entre os sexos, pelo teste " $t$ " de Student, adotando-se $p<0,05$ como nível de significância.

A pesquisa foi submetida e aprovada pelo Comitê de Ética em Pesquisa da Universidade Estadual do Ceará (Protocolo n 033226630-3).

\section{RES U L T A D O S}

Entre os indivíduos obesos pesquisados, 49 $(61,2 \%)$ pertenciam ao sexo feminino e 31 $(38,8 \%)$ ao sexo masculino. A Tabela 1 exibe a distribuição do grupo segundo sexo e faixa etária. A idade dos indivíduos variou de 20 a 83 anos, com média de 46,9 anos, tendo as mulheres apresentado média de idade de 49,6 anos (20 a 83 anos), um pouco superior à dos homens, de 42,6 anos (21 a 75 anos). A maioria dos indivíduos esteve na faixa etária de 40 anos ou mais (62,5\%), com uma maior concentração entre 50 e 59 anos $(26,2 \%)$.

A distribuição dos pacientes conforme o grau de obesidade e o sexo mostrou haver um maior percentual de pessoas com obesidade 
Tabela 1. Distribuição dos indivíduos obesos ( $n=80$ ) segundo faixa etária e sexo. Fortaleza, CE, 2004.

\begin{tabular}{|c|c|c|c|c|c|c|}
\hline \multirow{3}{*}{ Faixa etária (anos) } & \multicolumn{6}{|c|}{ Sexo } \\
\hline & \multicolumn{2}{|c|}{ Masculino $(n=31)$} & \multicolumn{2}{|c|}{ Feminino $(n=49)$} & \multicolumn{2}{|c|}{ Total } \\
\hline & $\mathrm{n}$ & $\%$ & $\mathrm{n}$ & $\%$ & $n$ & $\%$ \\
\hline $20-29$ & 5 & 16,1 & 5 & 10,2 & 10 & 12,5 \\
\hline 30-39 & 10 & 32,3 & 10 & 20,4 & 20 & 25,0 \\
\hline $40-49$ & 5 & 16,1 & 8 & 16,3 & 13 & 16,3 \\
\hline $50-59$ & 9 & 29,1 & 12 & 24,5 & 21 & 26,2 \\
\hline $60-69$ & 1 & 3,2 & 9 & 18,4 & 10 & 12,5 \\
\hline$\geq 70$ & 1 & 3,2 & 5 & 10,2 & 6 & 7,5 \\
\hline Total & 31 & 100,0 & 49 & 100,0 & 80 & 100,0 \\
\hline
\end{tabular}

grau I (77,5\%), como exposto na Tabela 2. Essa distribuição refletiu-se nas médias de Índice de Massa Corporal, seja para o grupo como um todo - 33,6 $(30,0-44,5) \mathrm{kg} / \mathrm{m}^{2}$ - ou para quaisquer dos sexos - 33,4 $(30,1-43,9) \mathrm{kg} / \mathrm{m}^{2}$ para o sexo masculino e $33,7(30,0-44,5) \mathrm{kg} / \mathrm{m}^{2}$ para o sexo feminino. Houve um predomínio acentuado de obesidade grau I entre os homens $(87,0 \%)$, enquanto entre as mulheres, apesar da maior proporção também de obesidade grau I $(71,4 \%)$, houve uma considerável presença de obesidade grau II (22,5\%).

Foi baixa a variedade de alimentos consumidos por, pelo menos, $50 \%$ dos indivíduos. Do grupo de laticínios, apenas leite de vaca (integral ou desnatado) e queijo foram citados, respectivamente por $65,0 \%$ e $73,8 \%$ dos entrevistados. Dentre frutas e hortaliças foram referidos: alface $(61,3 \%)$, cenoura $(60,0 \%)$, batata inglesa $(53,8 \%)$, tomate $(53,8 \%)$, banana $(50,0 \%)$ e mamão (50,0\%). Do grupo de carnes em geral houve citação apenas de frango $(86,3 \%)$ e de carne bovina $(82,5 \%)$. Como cereais e leguminosas, referência a arroz (88,8\%), pão francês $(78,8 \%)$ e feijão $(72,5 \%)$. Do grupo dos açúcares houve citação apenas de açúcar refinado $(62,5 \%)$ e, do grupo de óleos e gorduras, apenas óleo de soja $(100,0 \%)$. Entre os diversos, foi citado o café $(73,8 \%)$ e refrigerante $(50,0 \%)$.

Na Tabela 3 as refeições das dietas ingeridas estão categorizadas conforme o índice glicêmico. Pôde-se observar que o desjejum mostrou-se adequado apenas para um percentual

Tabela 2. Distribuição dos indivíduos obesos segundo grau de obesidade* e sexo. Fortaleza, CE, 2004

\begin{tabular}{|c|c|c|c|c|c|c|}
\hline \multirow{3}{*}{ IMC } & \multicolumn{6}{|c|}{ Sexo } \\
\hline & \multicolumn{2}{|c|}{ Masculino } & \multicolumn{2}{|c|}{ Feminino } & \multicolumn{2}{|c|}{ Total } \\
\hline & $n$ & $\%$ & $n$ & $\%$ & $n$ & $\%$ \\
\hline Obesidade grau I & 27 & 87,0 & 35 & 71,4 & 62 & 77,5 \\
\hline Obesidade grau II & 2 & 6,5 & 11 & 22,5 & 13 & 16,3 \\
\hline Obesidade grau III & 2 & 6,5 & 3 & 6,1 & 5 & 6,2 \\
\hline Total & 31 & 100,0 & 49 & 100,0 & 80 & 100,0 \\
\hline
\end{tabular}

*Determinado segundo o índice de massa corporal (IMC). WHO ${ }^{8}$.

Tabela 3. Distribuição dos indivíduos obesos segundo sexo e índice glicêmico das refeições consumidas. Fortaleza, CE, 2004.

\begin{tabular}{|c|c|c|c|c|c|c|c|c|c|c|c|c|}
\hline \multirow{3}{*}{$\begin{array}{l}\text { Índices } \\
\text { glicêminco }\end{array}$} & \multicolumn{12}{|c|}{ Refeições } \\
\hline & \multicolumn{2}{|c|}{ Desjejum } & \multicolumn{2}{|c|}{ Lanche matutino } & \multicolumn{2}{|c|}{ Almoço } & \multicolumn{2}{|c|}{ Lanche vespertino } & \multicolumn{2}{|c|}{ Jantar } & \multicolumn{2}{|c|}{ Ceia } \\
\hline & $n$ & $\%$ & $n$ & $\%$ & $\mathrm{n}$ & $\%$ & $n$ & $\%$ & $\mathrm{n}$ & $\%$ & $\mathrm{n}$ & $\%$ \\
\hline Baixo & 13 & 17,1 & 29 & 55,8 & 53 & 68,8 & 24 & 40,0 & 28 & 35,4 & 22 & 57,9 \\
\hline Moderado & 37 & 48,7 & 20 & 38,4 & 24 & 31,2 & 21 & 35,0 & 32 & 40,5 & 12 & 31,6 \\
\hline Alto & 26 & 34,2 & 3 & 5,8 & 0 & 0 & 15 & 25,0 & 19 & 24,1 & 4 & 10,5 \\
\hline Total & $76^{a}$ & 100,0 & $52^{a}$ & 100,0 & $77^{a}$ & 100,0 & $60^{a}$ & 100,0 & $79^{a}$ & 100,0 & $38^{\mathrm{a}}$ & 100,0 \\
\hline
\end{tabular}

a número de indivíduos que consomem a respectiva refeição. 
pequeno de indivíduos (17,1\%). Em comparação às demais, esta foi a refeição do dia com maiores porcentagens de moderado e alto IG. Os alimentos que mais freqüentemente contribuíram para a elevação do IG desta refeição foram o pão e o açúcar refinado. Quando presentes, produtos lácteos, por terem IG baixo, ajudaram a reduzir o IG do desjejum.

Quanto ao lanche matutino, percebeu-se, nesta refeição, maior prevalência de baixo IG, o que pode ser atribuído à presença de frutas ou devido à mesma ter se constituído apenas de café. Porém, quando houve o consumo de açúcar refinado, o IG da refeição elevou-se, tornando-se predominantemente moderado.

O almoço foi a refeição mais adequada, não sendo verificada, inclusive, nenhuma dieta de alto IG. A presença de hortaliças (apesar de algumas não serem consumidas por, ao menos, $50 \%$ dos indivíduos) nesta refeição, bem como do feijão, ajudou a reduzir a contribuição dos alimentos de alto IG, como arroz, batata e refrigerante, para o IG final da refeição.

Verifica-se o predomínio de lanches vespertinos de IG inadequado (moderado e alto). Aí pode ter havido influência da combinação do consumo de café adoçado com açúcar refinado e pão francês. À semelhança do desjejum e do lanche matutino, a presença de frutas e produtos lácteos manteve o IG mais baixo.

Quanto ao jantar, em oposição ao almoço, foi, predominantemente, inadequado. A ocorrência freqüente de lanches substituindo uma refeição tradicional teve parcela de participação considerável ao IG do jantar. Assim, foi comum encontrar, mais uma vez, o pão francês (alto IG) como grande contribuinte para o alto IG da refeição, quando associado ao refrigerante ou ao café, ou café com leite adoçado com açúcar refinado. Quando o jantar tinha característica de almoço, a refeição, geralmente, resultou em baixo índice glicêmico.

A ceia foi a refeição com maior percentual de abstinência, não sendo realizada por mais da metade dos pacientes $(52,5 \%)$. Contrariamente ao jantar, esta refeição mostrou-se adequada quanto ao IG. A maior prevalência de baixo índice glicêmico deveu-se, predominantemente, ao consumo de ceias à base de frutas e/ou produtos lácteos.

Baseado na Tabela 4, pode-se afirmar que o índice glicêmico global das dietas confirma tal inadequação, com apenas pouco mais de $21 \%$ dos obesos consumindo dietas de baixo índice glicêmico e prevalecendo dietas de IG moderado, como confirmado pela média de índice glicêmico das dietas desta população, que foi 59,23 (desvio-padrão - $\mathrm{DP}=7,32$ ). Entre os homens a média foi de 59,43 ( $D P=6,96)$ e entre as mulheres $59,09(\mathrm{DP}=7,60)$, sem diferença estatística (teste " $t$ " $=0,209 ; p=0,835$ ).

A carga glicêmica diária, que traduz a quantidade e a qualidade dos carboidratos consumidos, mostrou-se mais insatisfatória ainda que o índice glicêmico das dietas, pois $91,2 \%$ das mesmas mostraram-se inadequadas. A CG foi mais

Tabela 4. Distribuição dos indivíduos obesos segundo categorias de índice glicêmico (IG) e carga glicêmica (CG) dietéticos globais e sexo. Fortaleza, CE, 2004

\begin{tabular}{|c|c|c|c|c|c|c|c|c|c|c|c|c|}
\hline \multirow{4}{*}{ Categorias } & \multicolumn{12}{|c|}{ Sexo } \\
\hline & \multicolumn{4}{|c|}{ Masculino (M) } & \multicolumn{4}{|c|}{ Feminino (F) } & \multicolumn{4}{|c|}{ Total } \\
\hline & \multicolumn{2}{|c|}{ IG } & \multicolumn{2}{|c|}{ CG } & \multicolumn{2}{|c|}{ IG } & \multicolumn{2}{|c|}{$C G$} & \multicolumn{2}{|c|}{ IG } & \multicolumn{2}{|c|}{$C G$} \\
\hline & $\mathrm{n}$ & $\%$ & $\mathrm{n}$ & $\%$ & $\mathrm{n}$ & $\%$ & $\mathrm{n}$ & $\%$ & $\mathrm{n}$ & $\%$ & $n$ & $\%$ \\
\hline Baixo & 4 & 12,9 & 1 & 3,2 & 13 & 26,5 & 6 & 12,2 & 17 & 21,3 & 7 & 8,8 \\
\hline Moderado & 26 & 83,9 & 9 & 29,0 & 32 & 65,3 & 17 & 34,7 & 58 & 72,5 & 26 & 32,5 \\
\hline Alto & 1 & 3,2 & 21 & 67,8 & 4 & 8,2 & 26 & 53,1 & 5 & 6,2 & 47 & 58,7 \\
\hline Total & 31 & 100,0 & 31 & 100,0 & 49 & 100,0 & 49 & 100,0 & 80 & 100,0 & 80 & 100,0 \\
\hline
\end{tabular}

IG médio - M: 59,43; F: 59,09 - t=0,209; $p=0,835 ;$ CG média - M: 165,37; F: 130,15 - $t=2,543 ; p=0,014$. 
inadequada para os homens $(t=2,543 ; p=0,014)$. A CG média da dieta do sexo masculino foi 165,37 $(D P=67,40)$ e do feminino $130,15(D P=47,08)$.

O índice glicêmico e a carga glicêmica diários foram confrontados e mostraram correlação estatística diretamente proporcional tanto para o grupo em geral, quanto para ambos os sexos (IG geral x CG geral: $r=0,405$ e $p<0,0001$; IG masculino $\times$ CG masculino $r=0,453$ e $p=0,010$; IG feminino $\times$ CG feminino: $r=0,406$ e $p=0,004$ ).

Não houve correlação entre IMC e IG (IMC geral x IG geral: $r=-0,018$ e $p=0,874$; IMC masculino x IG masculino: $r=-0,238$ e $p=0,198$; IMC feminino $x$ IG feminino: $r=0,101$ e $p=0,489$ ). O mesmo ocorreu ao investigar correlação entre IMC e CG (IMC geral x CG geral: $r=0,033$ e $p=0,772$; IMC masculino x CG masculino: $r=-0,157$ e $p=0,399$; IMC feminino $\times C G$ feminino: $r=0,221$ e $p=0,127$ ).

A média de consumo energético diário da população foi de $2148,28 \mathrm{kcal}(1107,95$ - 6246,75 kcal). Entre os homens, a média foi maior que entre as mulheres, isto é, $2503,92 \mathrm{kcal}$ (1494,58 - 6246,75kcal) versus $1937,79 \mathrm{kcal}$ $(1107,95$ - 4869,93kcal), respectivamente $(\mathrm{t}=3,589 ; p=0,001)$.

A ingestão energética do grupo correlacionou-se de forma diretamente proporcional com a carga glicêmica $(r=0,708 ; p=0,000)$, mas não houve correlação com o IG $(r=0,030$; $p=0,789$ ). Considerando os sexos, a situação se reproduziu tanto entre as mulheres (VCT x IG: $r=-0,051, p=0,729$; VCT $\times$ CG: $r=0,686$, $p<0,0001)$, como entre os homens (VCT $\times$ IG: $r=0,110, p=0,556$; VCT $\times$ CG: $r=0,663, p<0,0001)$.

\section{DIS CUSS Ã O}

O maior contingente feminino encontrado na presente pesquisa é condizente com relatos de Matos \& Zanella ${ }^{16}$, de que as mulheres representam a maior demanda dos serviços destinados a controle da obesidade.

Relativamente ao índice glicêmico, em estudo que confrontou o IG de refeições de funcionários hipertensos e normotensos da Universidade Estadual do Ceará, Almeida ${ }^{17}$ encontrou como sendo constituídos de índice glicêmico inadequado, em ambos os grupos, o desjejum e o jantar, duas das três refeições também consideradas de IG insatisfatório nesta pesquisa. Similarmente ao presente estudo, pão francês no desjejum, batata e arroz combinados no almoço, pão francês com café no lanche vespertino, lanches no jantar e pão isolado ou juntamente com biscoitos, ou ainda combinado com sucos ou refrigerantes na ceia, mostraram-se contribuintes com a constituição de um IG inadequado. Da mesma forma, frutas nos lanches e na ceia, sucos de frutas no lanche da manhã e leite na ceia foram vistos como interferentes na redução do índice glicêmico. A concordância acerca desses achados, nos dois estudos, talvez possa sugerir pontos estratégicos da dieta para intervenção em planejamentos dietéticos com vistas a reduzir o índice glicêmico consumido.

Os pães têm amido totalmente gelatinizado, portanto, um alto índice glicêmico, em decorrência da alta temperatura de cocção e da mistura excessiva a que são submetidos durante o processamento ${ }^{18}$.

Os produtos lácteos colaboram com a redução de efeitos hiperglicêmicos da dieta ${ }^{18}$. 0 leite integral, especificamente, possui como açúcar natural a lactose, um dissacarídeo que deve ser digerido em seus açúcares componentes antes da absorção. Os dois açúcares resultantes, glicose e galactose, competem entre si pela absorção. Assim, além de a proteína e a gordura do leite reduzirem o IG desse alimento, a competição entre estes monossacarídeos retarda a absorção e também diminui o índice glicêmico ${ }^{13}$.

O arroz é um alimento com baixo teor de amilose e com muita amilopectina, sendo seu amido altamente gelatinizado durante o cozimento e, portanto, facilmente decomposto por enzimas digestivas, daí seu IG moderado ${ }^{13}$.

Para Brand-Miller et al. ${ }^{13}$, a maioria das frutas tem baixo IG e confere o referido efeito redutor no índice glicêmico das refeições devido 
ao seu alto teor de fibras, embora isso não possa ser generalizado para todos os componentes desse grupo de alimentos.

Como referido nos resultados, encontrou-se média de IG diário de 59,23. Esse valor, quando multiplicado por 1,43 para ser convertido no índice glicêmico correspondente ao padrão pão branco, procedimento utilizado por Ma et al. ${ }^{19}$, resulta em 84,69 . Tal valor é semelhante ao achado desses autores, que também se basearam em recordatórios habituais de 7 dias, embora o estudo não tenha sido realizado com obesos, mas com indivíduos com sobrepeso, em Massachussets, detectando que o IG médio das dietas consumidas pela população foi de 81,7 . Liu et al..$^{20}$ encontraram média de IG inferior à do presente estudo, de 75 (padrão pão branco), em pesquisa realizada com 185 mulheres saudáveis em pré-menopausa, eutróficas ou não.

Não pode deixar de ser comentado que todos os estudos que trabalham com informações obtidas a partir de recordatórios possuem desvantagens, pois dependem de treinamento especializado do entrevistador e da memória do entrevistado ${ }^{9}$. No entanto, o profissional responsável pela obtenção das informações do serviço avaliado era nutricionista, com especialidade na área clínica, o que contorna a questão do treinamento especializado, pois metodologias de inquéritos dietéticos são extensivamente debatidas e operacionalizadas em cursos de graduação em Nutrição, e o protocolo adotado pelo serviço é respaldado como recomendado para a prática clínica ${ }^{9}$. De qualquer forma, é sempre apropriado que se busque, na literatura, comparações com estudos metodologicamente semelhantes, que é o caso da pesquisa conduzida por Ma et al. ${ }^{19}$.

No que tange à CG, comparando a média obtida com a da população do estudo de Ma et al. ${ }^{19}$, os resultados da presente pesquisa o superam, ou seja, 197,8 de Ma et al. ${ }^{19}$, utilizando pão branco como referência, versus 205,63 $(143,80 \times 1,43)$. A pesquisa supracitada, de Liu et al. ${ }^{20}$, também com pão branco como referência, encontrou média de carga glicêmica de 147, portanto, também inferior à do presente estudo.

O índice glicêmico e a carga glicêmica diários mostraram correlacionarem-se de maneira diretamente proporcional. Em concordância, Hodge et al. ${ }^{21}$, ao analisarem a associação entre IG, CG e diabetes a partir das dietas de 36.787 adultos de 40 a 69 anos, também encontraram correlação positiva, porém fraca, entre índice glicêmico e carga glicêmica.

Neste estudo, o IMC não mostrou associação alguma seja com o IG ou com a CG. O estudo de Ma et al. ${ }^{19}$, previamente citado, não detectou qualquer associação estatística significante quando o IMC foi relacionado com a CG, porém, contrapondo-se ao resultado aqui encontrado, índice glicêmico e índice de massa corporal foram diretamente associados. Em metanálise realizada, verificou-se relação significante e diretamente proporcional de ambas as variáveis com o IMC ${ }^{22}$. Como o presente estudo incluiu apenas 80 indivíduos, pode ter havido interferência do tamanho da amostra nos achados.

Mesmo não tendo sido observada qualquer correlação estatística do IG e da CG com o IMC, a ingestão energética mostrou relação diretamente proporcional com a carga glicêmica. Percebeu-se relativa escassez de estudos que observassem o comportamento do índice glicêmico e/ou carga glicêmica dietéticos de acordo com o VCT da dieta, não só em obesos, mas em grupos populacionais em geral. Em meio a essa escassez, Scaglioni et al. ${ }^{23}$ analisaram tal relação em dietas de 111 escolares (8 anos de idade - 105 eutróficos e 6 com sobrepeso). Semelhantemente à presente pesquisa, os autores afirmaram não encontrar, quanto ao índice glicêmico, nenhuma associação com a ingestão energética. Porém, detectaram associação positiva da carga glicêmica com a ingestão energética. Especificamente, o aumento da ingestão de massas, pão branco, batata cozida, produtos de confeitaria e doces mostrou ser um dos principais interferentes na elevação da CG.

Como a carga glicêmica relaciona tipo e quantidade de carboidrato consumido, os 
resultados fornecem indícios de que, mais que um aumento no consumo de carboidratos de IG inadequado, provavelmente ocorra entre os obesos uma ingestão de grandes porções de alimentos fontes de carboidratos disponíveis, sejam eles de baixo, médio ou alto IG, à medida que aumenta o VET. Estudos com amostras de base populacional talvez possam fornecer achados mais contundentes acerca dessas variáveis.

Os resultados deste estudo tendem a comprovar os relatos da literatura, de que existe algum tipo de inter-relação entre o índice glicêmico e/ou a carga glicêmica dietéticos e a obesidade. Apesar de a situação ponderal atual não estar associada ao IG ou à CG, pode ter havido influência progressiva desses dois fatores sobre o ganho de peso, pois o consumo energético dos indivíduos foi diretamente associado à carga glicêmica. Novamente, somente a realização de mais estudos possibilitará a confirmação ou não dessa hipótese.

Percebeu-se que a situação mostrou-se pior entre os homens, que ingeriram mais energia e tiveram CG dietética maior. Para o grupo estudado, portanto, sem pretensões de extrapolação, os homens requerem uma atenção mais diferenciada, além de parecer mais relevante uma preocupação com a carga glicêmica do que com oIG.

Assim, os achados apontam para a necessidade de instituir uma abordagem terapêutica junto aos indivíduos obesos avaliados, que inclua tanto a elaboração de uma prescrição dietética com menores quantidades de alimentos com alta CG e maiores quantidades de fontes alimentares de baixa $C G$, como a implantação de ações educativas que tornem esses indivíduos aptos a adotarem esse procedimento em longo prazo, mesmo quando tiverem alta do acompanhamento nutricional. Tal conduta é pertinente, principalmente, considerando que os alimentos amiláceos, em especial produtos de panificação, com alta CG, representam parte importante da alimentação dessa população.

Em conclusão, parece claro que o cuidado nutricional dispensado a essa clientela terá maiores possibilidades de trazer efeitos benéficos à saúde, principalmente, quanto a evitar o agravamento do excesso de peso e de co-morbidades associadas à obesidade, bem como o desencadeamento destas últimas, se incluir a verificação e o manejo (se necessário) da CG no protocolo de atendimento.

\section{COLABORADORES}

H.A.C. SAMPAIO participou na coordenação geral, análise de dados e redação do artigo. B.Y.C. SILVA participou na tabulação, análise de dados e redação. M.O.D. SABRY participou na análise de dados e redação e P.C. ALMEIDA no tratamento estatístico e redação.

\section{REFERÊ N CIAS}

1. Saris WHM. Glycemic carbohydrate and body weight regulation. Nutr Rev. 2003; 61(5):10-6.

2. Menezes EW, Lajolo F. Índice glicêmico: critério de seleção de alimentos. Seminário Índice Glicémico en Salud y Alimentación Humana; 2002 Sep 12; San José, Costa Rica; 2002.

3. Ball SD, Keller KR, Moyer-Mileur LJ, Ding Y, Donaldson D, Jackson WD. Prolongation of satiety after low versus moderately high glycemic index meals in obese adolescents. Pediatrics. 2003; 111(3):488-94.

4. Food and Agriculture Organization. Carbohydrates in human nutrition: report of a joint FAOMHO expert consultation. Rome; 1997.

5. Ludwig DS. Dietary glycemic index and obesity. J Nutr. 2000; 130(Suppl 2):S280-3.

6. Bell SJ, Sears B. Low-glycemic load diets: impact on obesity and chronic diseases. Crit Rev Food Sci Nutr. 2003; 43(4):357-77.

7. Wolever TMS. Carbohidratos de digestión lenta: beneficios de una alimentación com bajo índice glicémico. Danone Nutritopics [periódico eletrônico] 2004 [acceso em 23 feb 2006]; (28). Disponible en: http://www.alimentariaonline.com/ apadmin/img/upload/MA003_diglentaglicemia WSF.pdf

8. World Health Organization. Obesity: preventing and managing the global epidemic - report of a WHO consultation on obesity. Switzerland (GE): WHO Library Cataloguing-in-Publication Data; 1997 Jun. Report No: 894. Sponsored by the World Health Organization. 
9. Fisberg RM, Martini LA, Slater B. Métodos de inquéritos alimentares. In: Fisberg RM, Slater B, Marchioni SML, Martini LA, editores. Inquéritos alimentares; métodos e bases científicas. Barueri: Manole; 2005.

10. Pinheiro ABV, Lacerda EMA, Benzecry EH, Gomes MCS, Costa VM. Tabela para avaliação de consumo alimentar em medidas caseiras. 4a. ed. São Paulo: Atheneu; 2002.

11. Foster-Powell K, Holt SHA, Brand-Miller JC. International table of glycemic index and glycemic load values: 2002. Am J Clin Nutr. 2002; 76(1): 5-56.

12. Brand-Miller JC, Burani J, Foster-Powell K, Holt S. The new glucose revolution: complete guide to glycemic index values. New York: Marlowe \& Company; 2003.

13. Brand-Miller JC, Foster-Powell K, Colagiuri S. A nova revolução da glicose. Rio de Janeiro: Elsevier; 2003.

14. Lau C, Faerch K, Glumer C, Tetens I, Pedersen O, Carstensen $B$, et al. Dietary glycemic index, glycemic load, fiber, simple sugars, and insulin resistance: the Inter99 study. Diabetes Care. 2005; 28: 1397-403.

15. Burani J. Gushers and tricklers: practical use of the glycemic index [cited 2007 Jul 22]. Available from: http://www.glycemicindex.com

16. Matos MIR., Zanella MT. Alterações do comportamento alimentar, ansiedade, depressão e imagem corporal em pacientes com obesidade grau III [cited 2006 Feb 2]. Available from: http:// www.abeso.org.br/revista/revista9/alteracoes.htm

17. Almeida DL. Índice glicêmico de dietas consumidas por indivíduos hipertensos e normotensos: fator de risco cardiovascular? [monografia de graduação]. Fortaleza: Universidade Estadual do Ceará; 2003.

18. Danone Vitapole. Food and Agriculture Organization. Glycaemic index and health: the quality of the evidence; 2001 Feb 21-23; Bandol, France. Paris: John Libbey Eurotext; 2001.

19. Ma Y, Olendzki B, Chiriboga D, Hebert JR, Li Y, Li $W$, et al. Association between dietary carbohydrates and body weight. Am J Epidemiol. 2005; 161(4):359-67.

20. Liu S, Manson JE, Stampfer MJ, Holmes MD, Hu FB, Hankinson FE, et al. Dietary glycemic load assessed by food-frequency questionnaire in relation to plasma high-density-lipoprotein cholesterol ond fasting plasma triacylglycerols in postmenopausal women. Am J Clin Nutr. 2001; 73(3):560-6.

21. Hodge AM, English DR, O'Dea K, Giles GG. Glycemic index and dietary fiber and the risk of type 2 diabetes. Diabetes Care. 2004; 27(11): 2701-6.

22. Brand-Miller JC, Holt SHA, Pawlak DB, McMillan J. Glycemic index and obesity. Am J Clin Nutr. 2002; 76(1 Suppl 2):281-5.

23. Scaglioni S, Stival G, Giovannini M. Dietary glycemic load, overall glycemic index, and serum insulin concentrations in healthy schoolchildren. Am J Clin Nutr. 2004; 79(2):339-40.

Recebido em: 10/10/2006

Versão final reapresentada em: 17/9/2007 Aprovado em: 10/10/2007 